\title{
Model Predictive Control of Linear Systems over Networks with State and Input Quantizations
}

\author{
Xiao-Ming Tang, Hong-Chun Qu, Hao-Fei Xie, and Ping Wang \\ Key Laboratory of Industrial Internet of Things \& Networked Control, Chongqing University of Posts and Telecommunications, \\ Ministry of Education, Chongqing 400065, China
}

Correspondence should be addressed to Xiao-Ming Tang; txmmyeye@126.com

Received 4 September 2013; Accepted 21 November 2013

Academic Editor: Linying Xiang

Copyright (C) 2013 Xiao-Ming Tang et al. This is an open access article distributed under the Creative Commons Attribution License, which permits unrestricted use, distribution, and reproduction in any medium, provided the original work is properly cited.

\begin{abstract}
Although there have been a lot of works about the synthesis and analysis of networked control systems (NCSs) with data quantization, most of the results are developed for the case of considering the quantizer only existing in one of the transmission links (either from the sensor to the controller link or from the controller to the actuator link). This paper investigates the synthesis approaches of model predictive control (MPC) for NCS subject to data quantizations in both links. Firstly, a novel model to describe the state and input quantizations of the NCS is addressed by extending the sector bound approach. Further, from the new model, two synthesis approaches of MPC are developed: one parameterizes the infinite horizon control moves into a single state feedback law and the other into a free control move followed by the single state feedback law. Finally, the stability results that explicitly consider the satisfaction of input and state constraints are presented. A numerical example is given to illustrate the effectiveness of the proposed MPC.
\end{abstract}

\section{Introduction}

Model predictive control (MPC) commonly refers to a class of computer control algorithms that use an explicit process model to forecast the future response of a plant [1]. In practical applications, MPC can now be found in a wide variety of industrial fields, such as chemical industries, food processing, and automotive applications, since it has advantages including good tracking performance, physical constraints handling, and extension to nonlinear systems. Not only that, in recent decades, it also has a great improvement in theoretical research; various methodologies have been addressed and developed, such as dynamic matrix control (DMC), generalized predictive control (GPC), and robust MPC. From the 1990s, the main stream of the theoretical research about MPC has been turned to the synthesis approach, which means that the MPC controller is synthesized so that the closed-loop system is stable whenever the optimization problem is feasible. A simple procedure of synthesis approach of MPC is to parameterize the infinite horizon control moves into a state feedback control law at each sampling time. In this field, [2] introduces the linear matrix inequality (LMI) technique to solve the optimization problem of minimizing an upper bound on "worst case" value of the infinite horizon objective function and reduces the problem to a convex optimization problem involving a set of LMIs. Reference [3] applies the technique in [2] off-line, and a sequence of control laws corresponding to a sequence of asymptotically stable invariant ellipsoids is constructed. The real-time state feedback gain is chosen from the designed control laws. In this way, the on-line computational burden can be significantly reduced. Another procedure of synthesis approach of MPC is to add free control moves before the linear state feedback law, instead of a single state feedback. In this field, the related works can be found in $[4,5]$. Reference [4] proposes a "quasi-min-max" MPC algorithm for linear parameter varying systems by adding one free control move, and the conservatism and feasibility in [2] are reduced and improved. Reference [5] extends the results in [4] to $N>1$ free control moves, which further improves the optimality and feasibility.

Networked control systems (NCSs) are distributed systems in which the sensors, the controllers, and the actuators are spatially distributed and interconnected through communication networks [6-12]. Data quantization is inevitable 


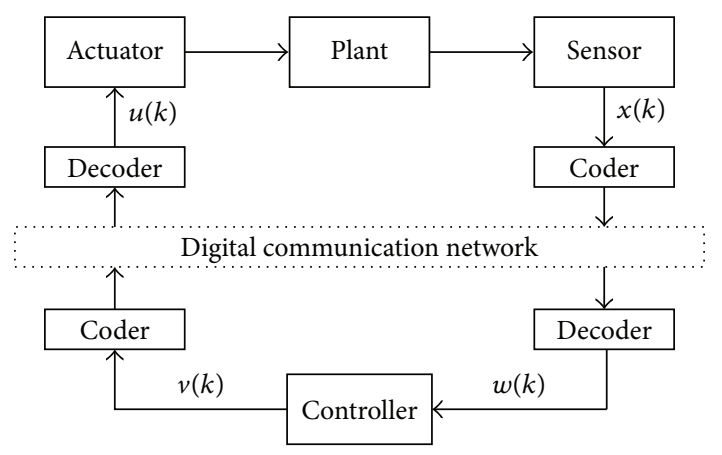

FIGURE 1: Structure of NCS with quantizations.

in NCSs, which is caused by $A / D$ and $D / A$ conversions and has an undesirable effect on system performance or even stability. The issue of mitigating the effect of data quantization has been considered by many researchers [1320]. For static and time-invariant quantizer, [13] first points out that the coarsest quantizer should follow a logarithmic law in quadratic stabilization of single-input-single-output (SISO) linear time-invariant (LTI) systems. Reference [14] generalizes the results of [13] to the multi-input-multi-output (MIMO) systems and the output feedback control and converts the quantized quadratic stabilization problem into the robust control problem by using the sector bound method. By adopting a quantization error dependent Lyapunov function, [15] proposes a new approach to the problem of analysis and synthesis for quantized feedback system based on the results in [14], which offers less conservative results. Based on the results in [14], a robust MPC strategy is proposed to deal with the stabilization of NCS with quantized control input in [16] and a simple dynamic scaling method for a logarithmic quantizer based output feedback controller is given in [17]. However, all the mentioned papers consider only one quantizer existing in one communication link, either in the sensor to controller (S-C) link or the controller to actuator (C-A) link. The quantized stabilization problem in both links has not received much attention than what it deserves. In the existing results, to the best of our knowledge, there are only a small amount of results [18-20] about the quantization problem when both the state and the input are quantized, and all of them do not consider the synthesis approach of MPC. This situation motivates our study presented here.

The synthesis approach of MPC is known as a control algorithm that can significantly improve the control performance, especially when system uncertainties exist; see $[2,3]$. Therefore, it is of theoretical and practical significance to extend these approaches to the networked control. However, very few works have focused on the synthesis approaches of MPC for NCS with quantizations. The goal of this paper is to give synthesis approaches of MPC for discrete LTI MIMO system over networks with state and input quantizations. Firstly, by applying the sector bound approach in [14], a novel model which describes the NCS with quantizers in both S-C and C-A links is established. The result converts the quantized stabilization problem into robust control problem. Secondly, based on the developed new model, two synthesis approaches of MPC, without free control move and with one free control move, are derived, respectively, by extending the results in $[2,4]$. Finally, the stability results of the closed-loop system are given by applying the Lyapunov method.

Notation. $I$ is the identity matrix with appropriate dimensions. For any vector $x$ and matrix $W,\|x\|_{W}^{2}:=x^{\mathrm{T}} W x$. The superscript $\mathrm{T}$ denotes the transpose for vectors or matrices. $x(k+i \mid k)$ is the value of vector $x$ at a future time $k+i$ predicted at time $k$.

\section{System Description}

Consider the quantized feedback control system in Figure 1. The plant to be controlled is modeled by

$$
x(k+1)=A(k) x(k)+B(k) u(k), \quad k \geq 0,
$$

where $u(k) \in \mathfrak{R}^{m}$ and $x(k) \in \Re^{n}$ are the control input and measurable state of the plant, respectively. $w(k) \in \mathfrak{R}^{n}$ and $v(k) \in \mathfrak{R}^{m}$ are input and output of the controller, respectively. We assume that $[A(k) \mid B(k)]$ varies inside a corresponding polytope $\Omega$ whose vertices consist of $L$ local systems matrices; that is,

$$
[A(k) \mid B(k)] \in \Omega, \quad k \geq 0,
$$

with

$$
\Omega=\operatorname{Co}\left\{\left[A_{1} \mid B_{1}\right],\left[A_{2} \mid B_{2}\right], \ldots,\left[A_{L} \mid B_{L}\right]\right\},
$$

where $\operatorname{Co}\{\cdot\}$ denotes the convex hull. The input and state constraints are

$$
-\underline{u} \leq u(k+i) \leq \bar{u}, \quad-\underline{\psi} \leq \Psi x(k+i+1) \leq \bar{\psi}, \quad \forall i \geq 0,
$$

where $\underline{u}:=\left[\underline{u}_{1}, \underline{u}_{2}, \ldots, \underline{u}_{m}\right]^{\mathrm{T}}, \bar{u}:=\left[\bar{u}_{1}, \bar{u}_{2}, \ldots, \bar{u}_{m}\right]^{\mathrm{T}}, \underline{u}_{j}>0$, $\bar{u}_{j}>0, j \in\{1,2, \ldots, m\} ; \underline{\psi}:=\left[\underline{\psi}_{1}, \underline{\psi}_{2}, \ldots, \underline{\psi}_{q}\right]^{\mathrm{T}}, \bar{\psi}:=$ $\left[\bar{\psi}_{1}, \bar{\psi}_{2}, \ldots, \bar{\psi}_{q}\right]^{\mathrm{T}}, \underline{\psi}_{s}>0, \bar{\psi}_{s}>0, s \in\{1,2, \ldots, q\} ; \Psi \in \mathfrak{R}^{q \times n}$.

The quantized state feedback controller is given by

$$
\begin{gathered}
u(k)=f(v(k)), \\
v(k)=K w(k)=K g(x(k)),
\end{gathered}
$$

where $K \in \Re^{m \times n}$ is the feedback gain to be designed and $f(\cdot)$ and $g(\cdot)$ are two static logarithmic quantizers which are defined as

$$
\begin{aligned}
& f(v(k))=\left[\begin{array}{llll}
f\left(v_{1}(k)\right) & f\left(v_{2}(k)\right) & \cdots & f\left(v_{m}(k)\right)
\end{array}\right]^{\mathrm{T}}, \\
& g(x(k))=\left[\begin{array}{llll}
g\left(x_{1}(k)\right) & g\left(x_{2}(k)\right) & \cdots & g\left(x_{n}(k)\right)
\end{array}\right]^{\mathrm{T}},
\end{aligned}
$$

where $f(\cdot)$ and $g(\cdot)$ are assumed to be symmetric, that is, $f\left(-v_{j}(k)\right)=-f\left(v_{j}(k)\right), j \in \mathscr{M}_{1}:=\{1,2, \ldots, m\}$, and $g\left(-x_{i}(k)\right)=-g\left(x_{i}(k)\right), i \in \mathscr{N}_{1}:=\{1,2, \ldots, n\}$, and $v_{j}(k)$ $\left(x_{i}(k)\right)$ is the $j$ th (ith) component of $v(k)(x(k))$. 
In terms of [14], the set of quantization levels for logarithmic quantizers $f(\cdot)$ and $g(\cdot)$ is described by

$$
\begin{array}{r}
\mathscr{U}_{\zeta}=\left\{ \pm u_{s}: u_{s}=\rho_{\zeta}^{s} u_{0}, s=0, \pm 1, \pm 2, \ldots\right\} \cup\{0\}, \\
0<\rho_{\zeta}<1, \quad u_{0}>0,
\end{array}
$$

where $\zeta$ is $f$ or $g$. The associated quantizer $\zeta$ is defined as follows:

$$
\zeta(\theta)= \begin{cases}u_{s}, & \text { if } \frac{1}{1+\delta_{\zeta}} u_{s}<\theta \leq \frac{1}{1-\delta_{\zeta}} u_{s}, \theta>0, \\ 0, & \text { if } \theta=0, \\ -\zeta(-\theta), & \text { if } \theta<0,\end{cases}
$$

with

$$
\delta_{\zeta}=\frac{1-\rho_{\zeta}}{1+\rho_{\zeta}}
$$

Denote by $\sharp g[\varepsilon]$ the number of quantization levels in the interval $[\varepsilon, 1 / \varepsilon]$. The density of the quantizer $\zeta$ is

$$
\vartheta_{\zeta}=\lim \sup _{\varepsilon \rightarrow 0} \frac{\# g_{\zeta}[\varepsilon]}{-\ln \varepsilon} \text {. }
$$

A small $\rho_{\zeta}$ means coarse quantization and a large $\rho_{\zeta}$ implies dense quantization. In the following, we will call $\rho_{\zeta}$ the quantization density of the quantizer $\zeta$, instead of $\vartheta_{\zeta}$, and assume that $\rho_{\zeta}$ is a known number and, hence, $\delta_{\zeta}$ is known and satisfies $0<\delta_{\zeta}<1$.

Applying the sector bound approach in [14], $f(v(k))$ and $g(x(k))$ can be written as

$$
\begin{aligned}
& f(v(k))=\left(I+\Delta_{f}(k)\right) v(k), \\
& g(x(k))=\left(I+\Delta_{g}(k)\right) x(k),
\end{aligned}
$$

where

$$
\begin{gathered}
\Delta_{f}(k)=\operatorname{diag}\left(\Delta_{f_{1}}(k), \Delta_{f_{2}}(k), \ldots, \Delta_{f_{m}}(k)\right), \\
\Delta_{g}(k)=\operatorname{diag}\left(\Delta_{g_{1}}(k), \Delta_{g_{2}}(k), \ldots, \Delta_{g_{n}}(k)\right)
\end{gathered}
$$

with $\left|\Delta_{f_{j}}(k)\right| \leq \delta_{f}, j \in \mathscr{M}_{1}$, and $\left|\Delta_{g_{i}}(k)\right| \leq \delta_{g}, i \in \mathcal{N}_{1}$.

Let $\mathscr{F}$ be the set of $m \times m$ diagonal matrices whose diagonal elements are either $-\delta_{f}$ or $\delta_{f}$. There are $2^{m}$ elements in $\mathscr{F}$. Suppose that each element in $\mathscr{F}$ is labeled as $\mathbb{F}_{j}, j \in$ $\mathscr{M}_{2}:=\left\{1,2, \ldots, 2^{m}\right\}$. Then, $\mathscr{F}=\left\{\mathbb{F}_{j}: j \in \mathscr{M}_{2}\right\}$. Let $\mathscr{G}$ be the set of $n \times n$ diagonal matrices whose diagonal elements are either $-\delta_{g}$ or $\delta_{g}$. There are $2^{n}$ elements in $\mathscr{G}$. Suppose that each element in $\mathscr{G}$ is labeled as $\mathbb{G}_{i}, i \in \mathcal{N}_{2}:=\left\{1,2, \ldots, 2^{n}\right\}$. Then, $\mathscr{G}=\left\{\mathbb{G}_{i}: i \in \mathcal{N}_{2}\right\}$. It is easy to show that

$$
\begin{gathered}
\Delta_{f}(k) \in \Omega^{f}=\operatorname{Co}\left\{\mathbb{F}_{j}: j \in \mathscr{M}_{2}\right\}, \\
\Delta_{g}(k) \in \Omega^{g}=\operatorname{Co}\left\{\mathbb{G}_{i}: i \in \mathscr{N}_{2}\right\} .
\end{gathered}
$$

This means that

$$
\Delta_{f}(k)=\sum_{j=1}^{2^{m}} \alpha_{j}(k)\left\{\mathbb{F}_{j}\right\}, \quad \Delta_{g}(k)=\sum_{i=1}^{2^{n}} \beta_{i}(k)\left\{\mathbb{G}_{i}\right\},
$$

where $\sum_{j=1}^{2^{m}} \alpha_{j}=1, \sum_{i=1}^{2^{n}} \beta_{i}=1, \alpha_{j} \geq 0, \beta_{i} \geq 0$.

Taking into account (11), the closed-loop system becomes

$$
\begin{aligned}
x(k+1)= & A(k) x(k) \\
& +B(k)\left(I+\Delta_{f}(k)\right) K\left(I+\Delta_{g}(k)\right) x(k) .
\end{aligned}
$$

It is shown in [14] that the system (15) and system (1), (2), (5) are equivalent for stability analysis.

\section{Stabilization Using Networked MPC}

3.1. Optimization Problem for Networked MPC. As in the nonnetworked MPC (see, e.g., [2, 4]), the state feedback $v=$ $K w$ will be utilized, where $K$ is to be optimized. We will give two different techniques: case 1 that is without free control move; case 2 that is with one free control move. For simplicity, denote

$$
\left(I+\Delta_{f}(k)\right) K(k)\left(I+\Delta_{g}(k)\right):=K(k)+\Delta K(k) .
$$

For case 1, the predicted control input becomes

$$
\begin{aligned}
u(k+l \mid k) & =f(g(x(k+l \mid k))) \\
& =(K(k)+\Delta K(k)) x(k+l \mid k), \quad l \geq 0 .
\end{aligned}
$$

The closed-loop state prediction satisfies

$$
\begin{aligned}
x(k+l+1 \mid k)= & A(k+l) x(k+l \mid k)+B(k+l) \\
& \times(K(k)+\Delta K(k)) x(k+l \mid k), \quad l \geq 0,
\end{aligned}
$$

where

$$
x(k \mid k) \in \partial(k) w(k)
$$

with

$$
\begin{gathered}
\partial(k)=\operatorname{diag}\left\{\partial_{1}(k), \partial_{2}(k), \ldots, \partial_{n}(k)\right\}, \\
\left(1+\delta_{g}\right)^{-1} \leq \partial_{i}(k) \leq\left(1-\delta_{g}\right)^{-1}, \quad i \in \mathcal{N}_{1} .
\end{gathered}
$$

Let $\mathscr{C}=\left\{\mathbb{C}_{h}: h \in \mathcal{N}_{2}\right\}$ be the set of $n \times n$ diagonal matrices whose diagonal elements are either $\left(1+\delta_{g}\right)^{-1}$ or $\left(1-\delta_{g}\right)^{-1}$. Then, it is shown that $\partial(k) \in \Omega^{c}=\operatorname{Co}\left\{\mathbb{C}_{h}: h \in \mathcal{N}_{2}\right\}$.

The objective of case 1 is to design $K(k)$ which drives (18) to the equilibrium point $x=0$, by minimizing the infinite horizon worst case performance index at each sampling instant:

$$
\min _{K(k)} \max _{\star} J(k)=\sum_{l=0}^{\infty}\left[\|x(k+l \mid k)\|_{\mathscr{Q}}^{2}+\|u(k+l \mid k)\|_{\mathscr{R}}^{2}\right]
$$

s.t. $\quad(4),(18),(19)$, 
where $\star=\left\{\partial(k) \in \Omega^{c}, \Delta_{f}(k) \in \Omega^{f}, \Delta_{g}(k) \in \Omega^{g},[A(k+l) \mid\right.$ $B(k+l)] \in \Omega, l \geq 0\} ; \mathbb{Q}$ and $\mathscr{R}$ are symmetric positive-definite weight matrices.

For case 2, the output of the controller achieves

$$
\begin{gathered}
v(k \mid k)=v(k), \quad l=0, \\
v(k+l \mid k)=(k)\left(I+\Delta_{g}(k)\right) x(k+l \mid k), \quad l \geq 1,
\end{gathered}
$$

where $v(k)$ is freedom for optimization. The predicted control input becomes

$$
\begin{aligned}
& u(k \mid k)=f(v(k \mid k))=\left(I+\Delta_{f}(k)\right) v(k), \quad l=0 \\
& u(k+l \mid k)=(K(k)+\Delta K(k)) x(k+l \mid k), \quad l \geq 1 .
\end{aligned}
$$

Hence, the closed-loop state prediction satisfies

$$
\begin{aligned}
x(k+1 \mid k)= & A(k) x(k \mid k) \\
& +B(k)\left(I+\Delta_{f}(k)\right) v(k), \quad l=0, \\
x(k+l+1 \mid k)= & A(k+l) x(k+l \mid k)+B(k+l) \\
& \times(K(k)+\Delta K(k)) x(k+l \mid k), \quad l \geq 1 .
\end{aligned}
$$

The objective of case 2 is to design $\{v(k), K(k)\}$ which drives (24) to the equilibrium point $x=0$, by minimizing the infinite horizon worst case performance index at each sampling instant:

$$
\begin{array}{ll}
\min _{K(k)} \max _{\star} & J(k), \\
\text { s.t. } & (4),(19),(24) .
\end{array}
$$

Problems (21) and (25) are the "min-max" optimization problem, for which the techniques of LMI can be efficiently utilized.

3.2. Networked MPC without Free Control Move. In order to derive an upper bound on $\max _{\star} J\left(j_{l}\right)$ and solve problem (21), let us impose the following stability constraint:

$$
\begin{aligned}
& V(x(k+l+1 \mid k))-V(x(k+l \mid k)) \\
& \quad \leq-\|x(k+l \mid k)\|_{\mathscr{Q}}^{2}-\|u(k+l \mid k)\|_{\mathscr{R}}^{2}, \quad \forall l \geq 0,
\end{aligned}
$$

where $V(x(k+l \mid k))=\|x(k+l \mid k)\|_{P}^{2}$. For stable closed-loop system, $\lim _{l \rightarrow \infty} V(x(k+l \mid k))=0$. Hence, by summing (26) from $l=0$ to $l=\infty$, we can obtain $V(x(k \mid k)) \geq J(k)$. Define $\mathrm{Q}=\gamma P^{-1}>0$, where $\gamma$ is a scalar. By minimizing $\gamma$ satisfying $V(x(k \mid k)) \leq \gamma$, the upper bound on performance cost is minimized. By using the Schur complement, $V(x(k \mid k)) \leq \gamma$ is guaranteed by

$$
\left[\begin{array}{cc}
1 & * \\
x(k \mid k) & Q
\end{array}\right] \geq 0 .
$$

Considering (19) and (14), this is equivalent to

$$
\left[\begin{array}{cc}
1 & * \\
\mathbb{C}_{h} w(k) & Q
\end{array}\right] \geq 0, \quad h \in \mathcal{N}_{2} .
$$

Substituting $Q=\gamma P^{-1}$ and using Schur complement, it is shown that (26) is equivalent to

$$
\left[\begin{array}{cccc}
Q^{-1} & * & * & * \\
A(k+l)+B(k+l)(K(k)+\Delta K(k)) & Q & * & * \\
Q^{1 / 2} & 0 & \gamma I & * \\
\mathscr{R}^{1 / 2}(K(k)+\Delta K(k)) & 0 & 0 & \gamma I
\end{array}\right] \geq 0 .
$$

Denote $K(k)=Y G^{-1}$ where $G$ is nonsingular matrix. Performing congruence transformation to (29) by $\operatorname{diag}\{G, I, I, I\}$, utilizing the fact that $G Q^{-1} G^{\mathrm{T}} \geq G+G^{\mathrm{T}}-Q$ and the convexity of polytopic description, it is shown that (29) can be guaranteed by

$$
\left[\begin{array}{cccc}
G+G^{\mathrm{T}}-Q & * & * & * \\
A_{s}+B_{s}\left(Y+\mathbb{F}_{j} Y+Y \mathbb{G}_{i}+\mathbb{F}_{j} Y \mathbb{G}_{i}\right) & Q & * & * \\
\mathbb{Q}^{1 / 2} G & 0 & \gamma I & * \\
\mathscr{R}^{1 / 2}\left(Y+\mathbb{F}_{j} Y+Y \mathbb{G}_{i}+\mathbb{F}_{j} Y \mathbb{G}_{i}\right) & 0 & 0 & \gamma I
\end{array}\right] \geq 0,
$$

Then, we consider handling of the input constraint. Define $\xi_{s}$ as the $s$ th row of the $m$-ordered identity matrix. We get

$$
\begin{aligned}
& \max _{l \geq 0}\left|\xi_{s} u(k+l \mid k)\right|^{2} \\
& \quad=\max _{l \geq 0}\left|\xi_{s}(K(k)+\Delta K(k)) x(k+l \mid k)\right|^{2} \\
& \quad \leq \max _{l \geq 0}\left\|\xi_{s}(K(k)+\Delta K(k)) Q^{1 / 2}\right\|^{2}\left\|Q^{-(1 / 2)} x(k+l \mid k)\right\|^{2} \\
& \quad \leq \max _{l \geq 0}\left\|\xi_{s}(K(k)+\Delta K(k)) Q^{1 / 2}\right\|^{2} .
\end{aligned}
$$

If there exists a symmetric matrix $Z, Z_{s s} \leq u_{s, \text { inf }}^{2}, s \in \mathbf{m}$, such that

$$
\max _{l \geq 0}(K(k)+\Delta K(k)) Q(K(k)+\Delta K(k))^{\mathrm{T}} \leq Z,
$$

then $\left|\xi_{s} u\left(k \mid j_{l}\right)\right| \leq u_{s, \text { inf }}, s \in \mathbf{m}$. It is easy to show that (32) is equivalent to

$$
\begin{aligned}
&\left(K(k)+\mathbb{F}_{j} K(k)+K(k) \mathbb{G}_{i}+\mathbb{F}_{j} K(k) \mathbb{G}_{i}\right) \\
& \times Q\left(K(k)+\mathbb{F}_{j} K(k)+K(k) \mathbb{G}_{i}+\mathbb{F}_{j} K(k) \mathbb{G}_{i}\right)^{\mathrm{T}} \leq Z, \\
& \quad j \in \mathscr{M}_{2}, \quad i \in \mathscr{N}_{2} .
\end{aligned}
$$

By performing congruence transformation to (33) by $\operatorname{diag}\{G, I\}$, utilizing the fact that $G Q^{-1} G^{\mathrm{T}} \geq G+G^{\mathrm{T}}-\mathrm{Q}$, and applying the Schur complement, it is shown that (33) can be guaranteed by

$$
\begin{gathered}
{\left[\begin{array}{cc}
G+G^{\mathrm{T}}-Q & * \\
Y+\mathbb{F}_{j} Y+Y \mathbb{G}_{i}+\mathbb{F}_{j} Y \mathbb{G}_{i} & Z
\end{array}\right] \geq 0, \quad j \in \mathscr{M}_{2}, i \in \mathcal{N}_{2},} \\
Z_{s s} \leq u_{s, \text { inf }}^{2}, \quad s \in \mathbf{m} .
\end{gathered}
$$


Lastly, consider the state constraint. Define $\xi_{s}$ as the sth row of the $q$-ordered identity matrix. Then, we have

$$
\begin{aligned}
& \max _{l \geq 0}\left|\xi_{s} \Psi x(k+l+1 \mid k)\right|^{2} \\
& \quad=\max _{l \geq 0}\left|\xi_{s} \Psi[\star] x(k+l \mid k)\right|^{2} \\
& \quad \leq \max _{l \geq 0}\left\|\xi_{s} \Psi[\star] Q^{1 / 2}\right\|^{2}\left\|Q^{-(1 / 2)} x(k+l \mid k)\right\|^{2} \\
& \quad \leq \max _{l \geq 0}\left\|\xi_{s} \Psi[\star] Q^{1 / 2}\right\|^{2},
\end{aligned}
$$

where $\star=A(k+l)+B(k+l)(K(k)+\Delta K(k))$. If there exists a symmetric matrix $\Gamma, \Gamma_{s s} \leq \psi_{s, \text { inf }}^{2}, s \in \mathbf{q}$, such that

$$
\max _{l \geq 0} \Psi \star Q \star{ }^{\mathrm{T}} \Psi^{\mathrm{T}} \leq \Gamma,
$$

then, by performing congruence transformation to (36) by $\operatorname{diag}\{G, I\}$, utilizing the fact that $G Q^{-1} G^{\mathrm{T}} \geq G+G^{\mathrm{T}}-Q$ and the convexity of polytopic description, and applying the Schur complement, it is easy to show that (36) is guaranteed by

$$
\begin{gathered}
{\left[\begin{array}{cc}
G+G^{\mathrm{T}}-Q & * \\
\Psi\left(A_{s} G+B_{s}\left(Y+\mathbb{F}_{j} Y+Y \mathbb{G}_{i}+\mathbb{F}_{j} Y \mathbb{G}_{i}\right)\right) & \Gamma
\end{array}\right] \geq 0} \\
s \in\{1,2, \ldots, L\}, \quad j \in \mathscr{M}_{2}, \quad i \in \mathcal{N}_{2} \\
\Gamma_{s s} \leq \psi_{s, \text { inf }}^{2}, \quad s \in \mathbf{q} .
\end{gathered}
$$

Therefore, the "min-max" optimization problem (21) for the proposed quantized state feedback MPC can be developed as

$$
\begin{array}{ll}
\min _{\gamma, Q, G, Y, \Gamma, Z} & \gamma \\
\text { s.t. } & (28),(30),(34), \text { and (37). }
\end{array}
$$

Theorem 1. If the optimization problem (38) is feasible at time $k$, then it is feasible for all times $t>k$, and the receding horizon quantized state feedback control move $u(k)=f(K(k) g(x(k)))$ asymptotically stabilizes the closed-loop system.

Proof. Firstly, we consider the recursive feasibility. Assume that the optimization problem (38) is feasible at time $k$. The only LMI in this problem which depends on the measured state of the system is (28). Hence, we only need to prove that (28) is feasible for future measured state $w(k+i), i>0$. From [2], when the constraints (28) and (30) are satisfied, it must have

$$
x(k+l \mid k)^{\mathrm{T}} \mathrm{Q}^{-1} x(k+l \mid k)<1, \quad l \geq 1 .
$$

At time $k$, the state prediction for $k+1$ is

$$
\begin{array}{r}
x(k+1 \mid k)=\left(A_{s}+B_{s}\left(K(k)+\mathbb{F}_{j} K(k)+K(k) \mathbb{G}_{i}\right.\right. \\
\left.\left.+\mathbb{F}_{j} K(k) \mathbb{G}_{i}\right)\right) \mathbb{C}_{h} w(k), \\
s \in\{1,2, \ldots, L\}, \quad j \in \mathscr{M}_{2}, i, h \in \mathscr{N}_{2} .
\end{array}
$$

At time $k+1$, there exist $[A \mid B] \in \Omega, \mathbb{F} \in \Omega^{f}, \mathbb{G} \in \Omega^{g}$, and $\mathbb{C} \in \Omega^{c}$ such that

$$
\begin{aligned}
x( & +1 \mid k+1) \\
& =(A+B(K(k)+\mathbb{F} K(k)+K(k) \mathbb{G}+\mathbb{F} K(k) \mathbb{G})) \mathbb{C} w(k) .
\end{aligned}
$$

From (40) and (41), it is easy to show that $x(k+1 \mid k+1)$ is contained in $x(k+1 \mid k)$. Taking into account (39), we get $x(k+l \mid k+1)^{\mathrm{T}} \mathrm{Q}^{-1} x(k+l \mid k+1)<1$, which is equivalent to

$$
\left[\begin{array}{cc}
1 & * \\
\mathbb{C}_{h} w(k+1) & Q
\end{array}\right] \geq 0, \quad h \in \mathcal{N}_{2} .
$$

Thus, the optimization problem is also feasible at time $k+1$, and the same results can be achieved at times $k+2, k+3, \ldots$ by analogy. Hence, the recursive feasibility of optimization problem (38) is guaranteed.

Secondly, we prove the asymptotic stability. Assume that the optimization problem (38) is feasible and the solution is depicted as “*”. By applying stability constraints, we have

$$
\begin{gathered}
\|x(k+1 \mid k)\|_{P^{*}(k)}^{2}-\|x(k \mid k)\|_{P^{*}(k)}^{2} \\
\leq-\|x(k \mid k)\|_{\mathscr{Q}}^{2}-\left\|u^{*}(k \mid k)\right\|_{\mathscr{R}}^{2} .
\end{gathered}
$$

Since $x(k+1 \mid k+1)$ is contained in $x(k+1 \mid k)$, the following holds:

$$
\begin{gathered}
\|x(k+1 \mid k+1)\|_{P^{*}(k)}^{2}-\|x(k \mid k)\|_{P^{*}(k)}^{2} \\
\leq-\|x(k \mid k)\|_{\mathscr{Q}}^{2}-\left\|u^{*}(k \mid k)\right\|_{\mathscr{R}}^{2} .
\end{gathered}
$$

By noting

$$
\begin{gathered}
\|x(k \mid k)\|_{P^{*}(k)}^{2} \leq \gamma^{*}(k), \\
\|x(k+1 \mid k+1)\|_{P(k+1)}^{2} \leq \gamma(k+1),
\end{gathered}
$$

it is admissible to choose

$$
\gamma(k+1)=\gamma^{*}(k)-\|x(k \mid k)\|_{\mathscr{Q}}^{2}-\left\|u^{*}(k \mid k)\right\|_{\mathscr{R}}^{2} .
$$

Solving the optimization problem (38) at time $k+1$, it leads to

$$
\gamma^{*}(k+1) \leq \gamma(k+1)
$$

It must have

$$
\gamma^{*}(k+1) \leq \gamma^{*}(k)-\|x(k \mid k)\|_{\mathscr{Q}}^{2}-\left\|u^{*}(k \mid k)\right\|_{\mathscr{R}}^{2} .
$$

Therefore, $\gamma^{*}(k)$ decreases monotonously and can be chosen as the Lyapunov function for proving the stability of the closed-loop system. 
3.3. Networked MPC with One Free Control Move. In Section 3.2, we developed the synthesis approach of networked MPC by parameterizing the infinite horizon control moves into a single state feedback law. This part, in order to reduce the conservatism, provides a synthesis approach of MPC by adding one free control move, which is easier to be feasible and can obtain more optimal control moves.

The optimization problem is shown in (25). Similar to Section 3.2, let us impose the stability constraint (26) for all $l \geq 1$. For stable closed-loop system, $\lim _{l \rightarrow \infty} V(x(k+l \mid$ $k))=0$. By summing (26) from $l=1$ to $l=\infty$, we get $J(k) \leq V(x(k+1 \mid k))+\|x(k \mid k)\|_{\mathscr{Q}}^{2}+\|u(k \mid k)\|_{\mathscr{R}}^{2}$. Let

$$
\begin{gathered}
V(x(k+1 \mid k)) \leq \gamma, \\
\|u(k \mid k)\|_{\mathscr{R}}^{2} \leq \gamma_{1},
\end{gathered}
$$

where $\gamma_{1}$ is a scalar. By defining $Q=\gamma P^{-1}>0$ and using Schur complement, it is shown that (49) is guaranteed by

$$
\left[\begin{array}{cc}
1 & * \\
x(k+1 \mid k) & Q
\end{array}\right] \geq 0 .
$$

Considering (14), (19) and (24), this is equivalent to

$$
\begin{gathered}
{\left[\begin{array}{cc}
1 & * \\
A_{s} C_{h} w(k)+B_{s}\left(I+\mathbb{F}_{j}\right) v(k) & Q
\end{array}\right] \geq 0} \\
s \in\{1,2, \ldots, L\}, \quad j \in \mathscr{M}_{2}, h \in \mathscr{N}_{2} .
\end{gathered}
$$

By considering (24) and (14), and applying Schur complement, it is shown that (50) is guaranteed by

$$
\left[\begin{array}{cc}
1 & * \\
\mathscr{R}^{1 / 2}\left(I+\mathbb{F}_{j}\right) v(k) & \gamma_{1} I
\end{array}\right] \geq 0, \quad j \in \mathscr{M}_{2} .
$$

Lemma 2. Suppose there exist a scalar $\gamma$, a vector $v(k)$, symmetric matrices $\Gamma, Z$, and $Q$, and any matrices $G, Y$ such that (30), (34), (37), (52), and the following are satisfied:

$$
\begin{gathered}
-\underline{u} \leq v(k) \leq \bar{u}, \\
-\underline{\psi} \leq \Psi x(k+1) \leq \bar{\psi} .
\end{gathered}
$$

Then, (4) is satisfied.

Proof. According to the deductions in Section 3.2, if (30) and (52) are satisfied, then (4) for $l \geq 1$ is guaranteed by (34) and (37). Equations (54) and (55) guarantee the satisfaction of (4) for $l=0$. This completes the proof.

According to (24), (55) is equivalent to

$$
-\underline{\psi} \leq A(k) x(k \mid k)+B(k)\left(I+\Delta_{f}(k)\right) v(k) \leq \bar{\psi} .
$$

Considering (19) and the convexity of the polytopic description, it is shown that (56) can be guaranteed by

$$
\begin{array}{r}
-\underline{\psi} \leq A_{s} C_{h} w(k)+B_{s}\left(I+\mathbb{F}_{j}\right) v(k) \leq \bar{\psi}, \\
s \in\{1,2, \ldots, L\}, \quad j \in \mathscr{M}_{2}, h \in \mathcal{N}_{2} .
\end{array}
$$

Therefore, the "min-max" optimization problem (25) for the proposed quantized networked MPC with one free control move can be approximated by

$$
\begin{array}{ll}
\min _{\gamma, \gamma_{1}, v(k), Q, G, Y, \Gamma, Z} & \gamma_{1}+\gamma \\
\text { s.t. } & (30),(34),(37),(52)-(54),(57) .
\end{array}
$$

Theorem 3. If the optimization problem (58) is feasible at time $k$, then it is feasible for all times $t>k$, and the receding horizon quantized state feedback control move $u(k)=f(v(x(k)))$ asymptotically stabilizes the closed-loop system.

Proof. Assume that the optimization problem (58) is feasible and the optimal solutions are depicted as “*."At time $k+1$, the feasible solution can be chosen as $v(k+1)=v^{*}(k+1 \mid k)=$ $K^{*}(k) w(k+1)$. Denote

$$
L(k)=\|x(k+1 \mid k)\|_{P(k)}^{2}+\|x(k \mid k)\|_{\mathscr{Q}}^{2}+\|u(k \mid k)\|_{\mathscr{R}}^{2} .
$$

We can take

$$
\begin{aligned}
L(k+1)= & \left\|x^{*}(k+1 \mid k)\right\|_{\mathscr{Q}}^{2}+\left\|u^{*}(k+1 \mid k)\right\|_{\mathscr{R}}^{2} \\
& +\left\|x^{*}(k+2 \mid k)\right\|_{P^{*}(k)}^{2} .
\end{aligned}
$$

It is easy to show

$$
J(k+1) \leq L(k+1) .
$$

Since stability constraint (26) is satisfied, it must have

$$
\begin{gathered}
\left\|x^{*}(k+2 \mid k)\right\|_{P^{*}(k)}^{2}-\left\|x^{*}(k+1 \mid k)\right\|_{P^{*}(k)}^{2} \\
\quad \leq-\left\|x^{*}(k+1 \mid k)\right\|_{\mathscr{Q}}^{2}-\left\|u^{*}(k+1 \mid k)\right\|_{\mathscr{R}}^{2} .
\end{gathered}
$$

Substituting (62) into (61), we get

$$
L(k+1) \leq\left\|x^{*}(k+1 \mid k)\right\|_{P^{*}(k)}^{2} \leq L^{*}(k) .
$$

At time $k+1$, the optimization is performed again, and it leads to

$$
L^{*}(k+1) \leq L(k+1)<L^{*}(k) .
$$

Hence, $L^{*}(k)$ is decreased monotonously, and we can choose it as the Lyapunov function for proving the stability of the closed-loop system. This completes the proof.

\section{Numerical Example}

In this section, the effectiveness of the proposed MPC is illustrated by a numerical example. Consider the following linear parameter varying system:

$$
\begin{aligned}
x(k+1)= & {\left[\begin{array}{cc}
0.872 & -0.0623 \kappa(k) \\
0.0935 & 0.997
\end{array}\right] x(k) } \\
& +\left[\begin{array}{c}
0.0935 \\
0.00478
\end{array}\right] u(k) .
\end{aligned}
$$




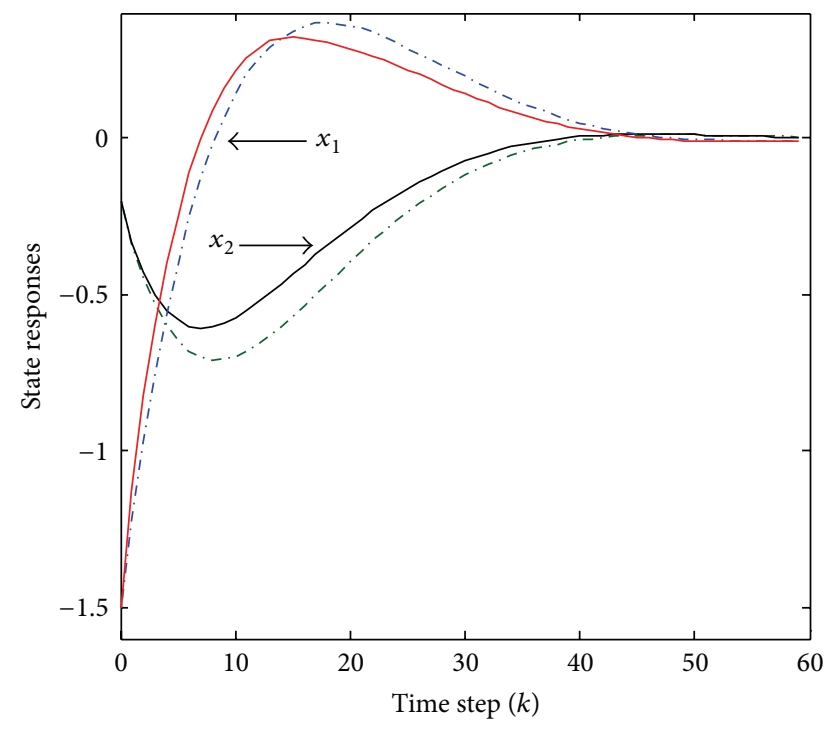

FIGURE 2: The state responses of the closed-loop system by applying (38) and (58).

We assume that the uncertain parameter $\kappa(k)$ lies in the area $\kappa(k) \in[1,5]$ and is generated by $\kappa(k)=1+4|\sin (0.01 k)|$. The initial state is assumed as $x(0)=\left[\begin{array}{ll}-1.5 & -0.2\end{array}\right]^{\mathrm{T}}$. The input constraint is $|u| \leq 1$. Moreover, for the networked environment, the quantizers $f(\cdot)$ and $g(\cdot)$ are chosen as logarithmic with quantization density $\rho_{f}=\rho_{g}=0.8091$ and the quantizer parameter $u_{0}$ is selected as $u_{0}=15$. It is easy to show that the set of quantization is able to cover the state trajectories according to the evaluation of the initial condition. The parameters of the cost function are $\mathscr{R}=1, \mathbb{Q}=$ I.

Let $J_{\text {true }}=\sum_{k=0}^{\infty}\left(x(k)^{\mathrm{T}} x(k)+u(k)^{2}\right)$ and denote $T_{\text {ave }}$ as the average computation time for each $k$. By solving (38) and (58), the resultant state trajectories, the control input, and the evolution of $\gamma(k)(L(k))$ are shown in Figures 2, 3, and 4, respectively, in dash lines (solid lines), with $J_{\text {true }}=$ 19.005 and $T_{\text {ave }}=0.2078 \mathrm{~s}\left(J_{\text {true }}=18.586\right.$ and $T_{\text {ave }}=$ $0.2954 \mathrm{~s})$. It is shown that the closed-loop system is stable by applying the two techniques we proposed. Compared with the networked MPC without free control move, by adding one free control move one can achieve faster state responses and more aggressive control moves. Moreover, $\gamma(k)$ and $L(k)$ are monotonic with the evolution of time.

\section{Conclusions}

In this paper, the synthesis approaches of MPC have been introduced to deal with the problem of NCS with input and state quantizations. The issue of quantized stabilization of this system is transformed into the robust control problem by applying the sector bound approach. Two synthesis approaches of MPC are formulated and the stability results for the closed-loop system are given by taking the presented

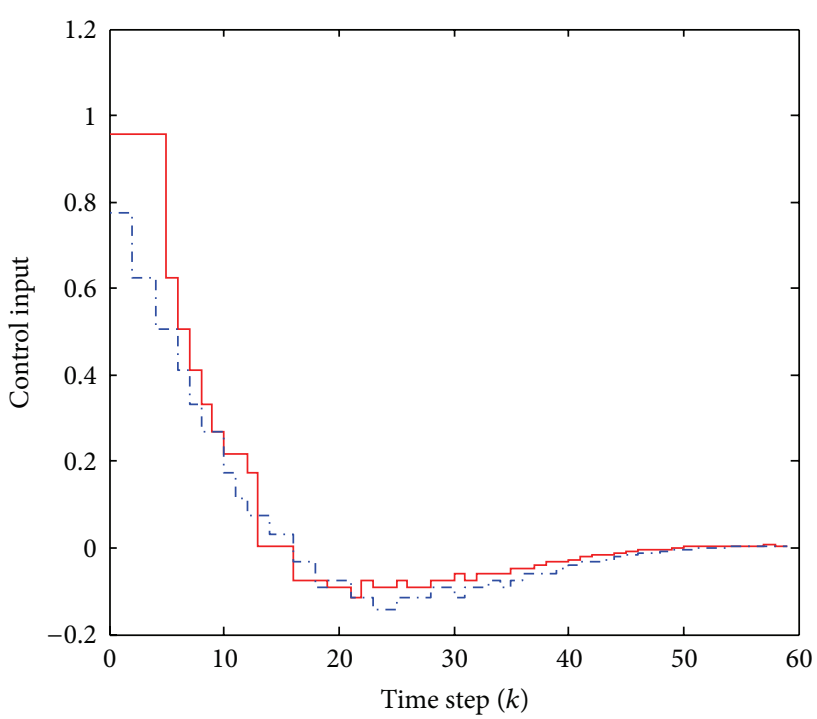

FIGURE 3: The control input by applying (38) and (58).

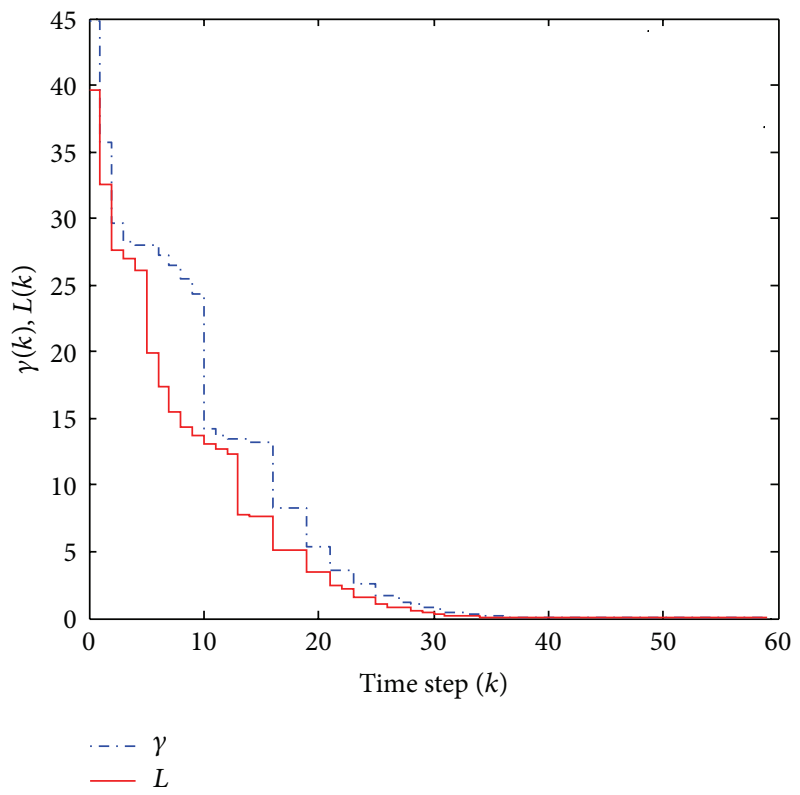

FIGURE 4: The evolutions of $\gamma(k)$ and $L(k)$ by applying (38) and (58), respectively.

MPC which explicitly consider the input and state constraints. The numerical example illustrates the effectiveness of the proposed techniques.

\section{Acknowledgments}

This work is supported by the National Natural Science Foundation of China (61102145, 61374093, and 61304197), the National Science and Technology Major Project (2013ZX03005005), the National Special Funds Program of Development of Internet of Things (2011.353), the Program of Development of Information Industry of Chongqing 
(2010.626), the Research Project of Chongqing Science and Technology Commission (cstc2013jcyjA40014), the Youth Science and Technology Innovation Talents Project of Chongqing Science and Technology Commission (cstc2013kjrcqnrc40005), the Scientific and Technological Research Program of Chongqing Municipal Education Commission (KJ130506, KJ130516), and the Doctoral Start-Up Fund of Chongqing University of Posts and Telecommunications (A2013-14).

\section{References}

[1] S. J. Qin and T. A. Badgwell, "A survey of industrial model predictive control technology," Control Engineering Practice, vol. 11, no. 7, pp. 733-764, 2003.

[2] M. V. Kothare, V. Balakrishnan, and M. Morari, "Robust constrained model predictive control using linear matrix inequalities," Automatica, vol. 32, no. 10, pp. 1361-1379, 1996.

[3] Z. Wan and M. V. Kothare, "An efficient off-line formulation of robust model predictive control using linear matrix inequalities," Automatica, vol. 39, no. 5, pp. 837-846, 2003.

[4] Y. Lu and Y. Arkun, "Quasi-Min-Max MPC algorithms for LPV systems," Automatica, vol. 36, no. 4, pp. 527-540, 2000.

[5] B. C. Ding, Y. G. Xi, and S. Y. Li, "A synthesis approach of online constrained robust model predictive control," Automatica, vol. 40, no. 1, pp. 163-167, 2004.

[6] W. Zhang, M. S. Branicky, and S. M. Phillips, "Stability of networked control systems," IEEE Control Systems Magazine, vol. 21, no. 1, pp. 84-99, 2001.

[7] G. Guo and Z. B. Lu, "Markov actuator assignment for networkedcontrol systems," European Journal of Control, vol. 18, no. 4, pp. 323-330, 2012.

[8] Y.-B. Zhao, G.-P. Liu, and D. Rees, "Design of a packetbased control framework for networked control systems," IEEE Transactions on Control Systems Technology, vol. 17, no. 4, pp. 859-865, 2009.

[9] Y.-B. Zhao, G. P. Liu, and D. Rees, "Improved predictive control approach to networked control systems," IET Control Theory and Applications, vol. 2, no. 8, pp. 675-681, 2008.

[10] J. Baillieul and P. J. Antsaklis, "Control and communication challenges in networked real-time systems," Proceedings of the IEEE, vol. 95, no. 1, pp. 9-28, 2007.

[11] J. Xiong and J. Lam, "Stabilization of linear systems over networks with bounded packet loss," Automatica, vol. 43, no. 1, pp. 80-87, 2007.

[12] T. M. Tang and B. C. Ding, "Model predictive control oflinear systems over networks with data quantizationsand packet losses," Automatica, vol. 49, no. 5, pp. 1333-1339, 2013.

[13] N. Elia and K. Mitter, "Stabilization of linear systems with limited information," IEEE Transactions on Automatic Control, vol. 46, no. 9, pp. 1384-1400, 2001.

[14] M. Fu and L. Xie, "The sector bound approach to quantized feedback control," IEEE Transactions on Automatic Control, vol. 50, no. 11, pp. 1698-1711, 2005.

[15] H. J. Gao and T. W. Chen, "A new approach to quantized feedback control systems," Automatica, vol. 44, no. 2, pp. 534$542,2008$.

[16] B. Xue, N. Li, S. Li, and Q. Zhu, "Robust model predictive control for networked control systems with quantisation," IET Control Theory and Applications, vol. 4, no. 12, pp. 2896-2906, 2010.
[17] M. Fu and L. Xie, "Finite-level quantized feedback control for linear systems," IEEE Transactions on Automatic Control, vol. 54, no. 5, pp. 1165-1170, 2009.

[18] D. Yue, C. Peng, and G. Y. Tang, "Guaranteed cost control of linear systems over networks with state and input quantisations," IEE Proceedings on Control Theory and Applications, vol. 153, no. 6, pp. 658-664, 2006.

[19] C. Peng and Y.-C. Tian, "Networked $H_{\infty}$ control of linear systems with state quantization," Information Sciences, vol. 177, no. 24, pp. 5763-5774, 2007.

[20] E. Tian, D. Yue, and C. Peng, "Quantized output feedback control for networked control systems," Information Sciences, vol. 178, no. 12, pp. 2734-2749, 2008. 


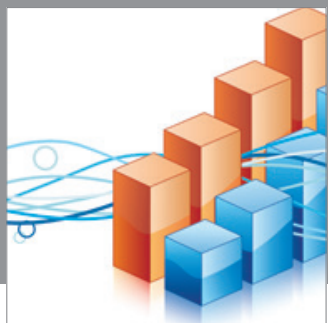

Advances in

Operations Research

mansans

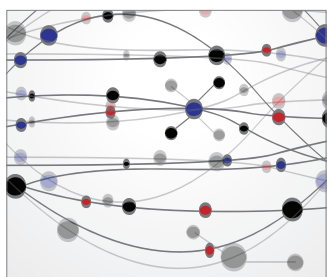

The Scientific World Journal
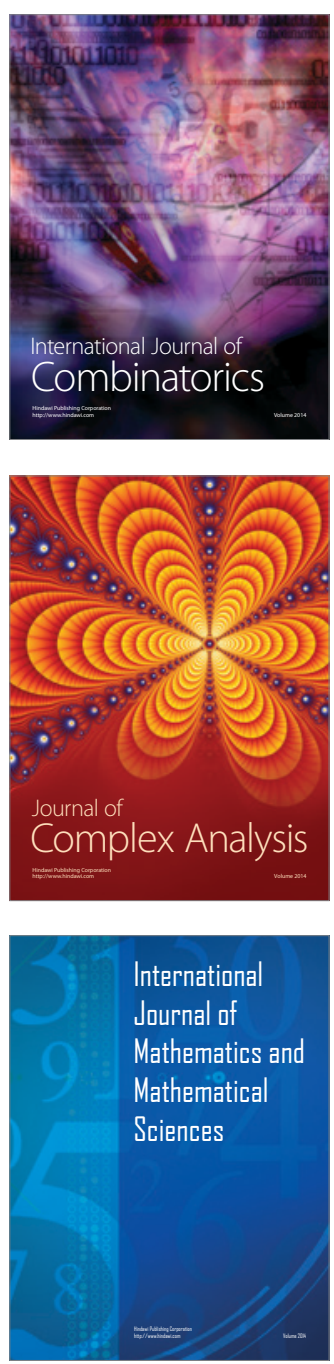
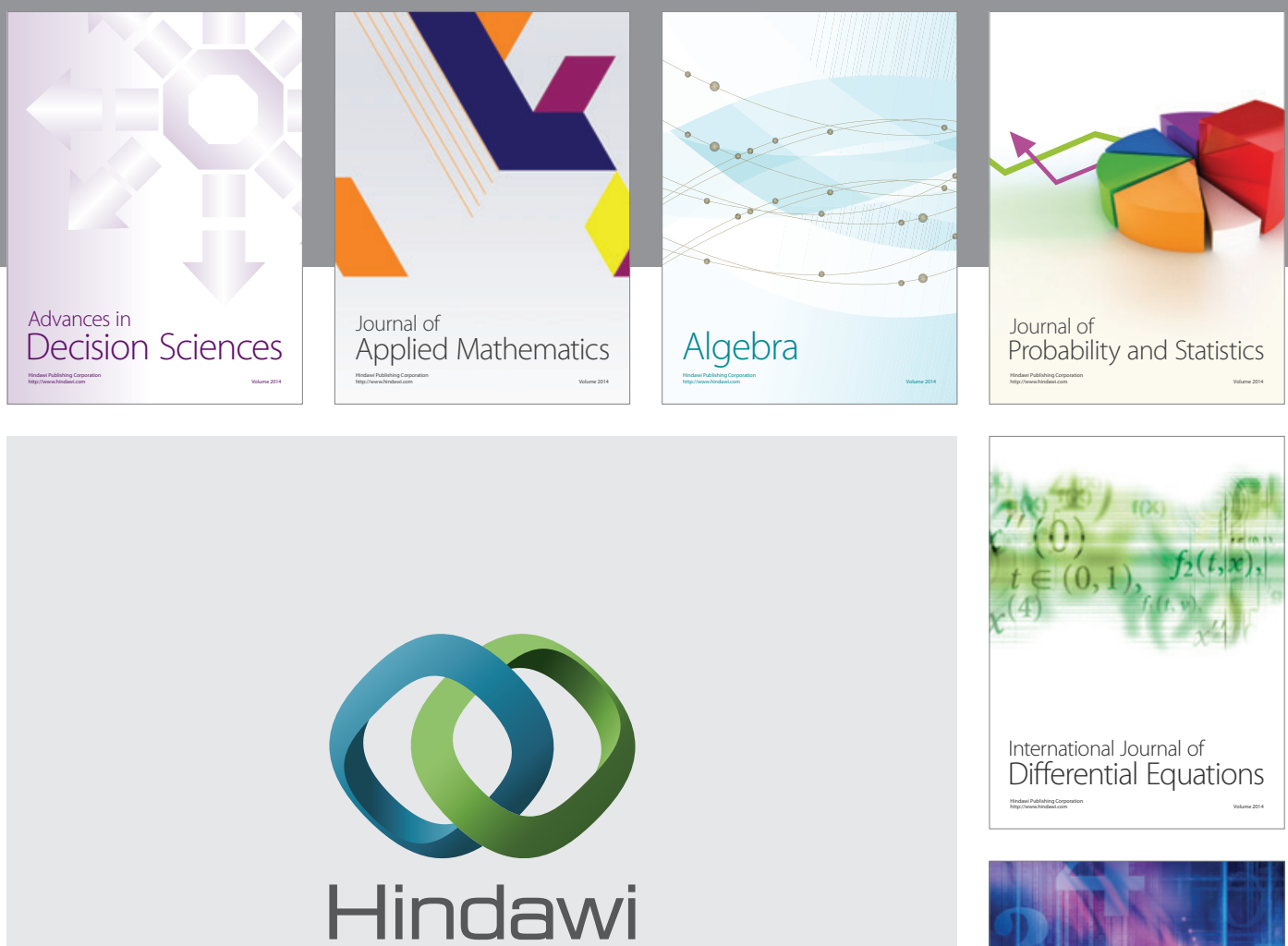

Submit your manuscripts at http://www.hindawi.com
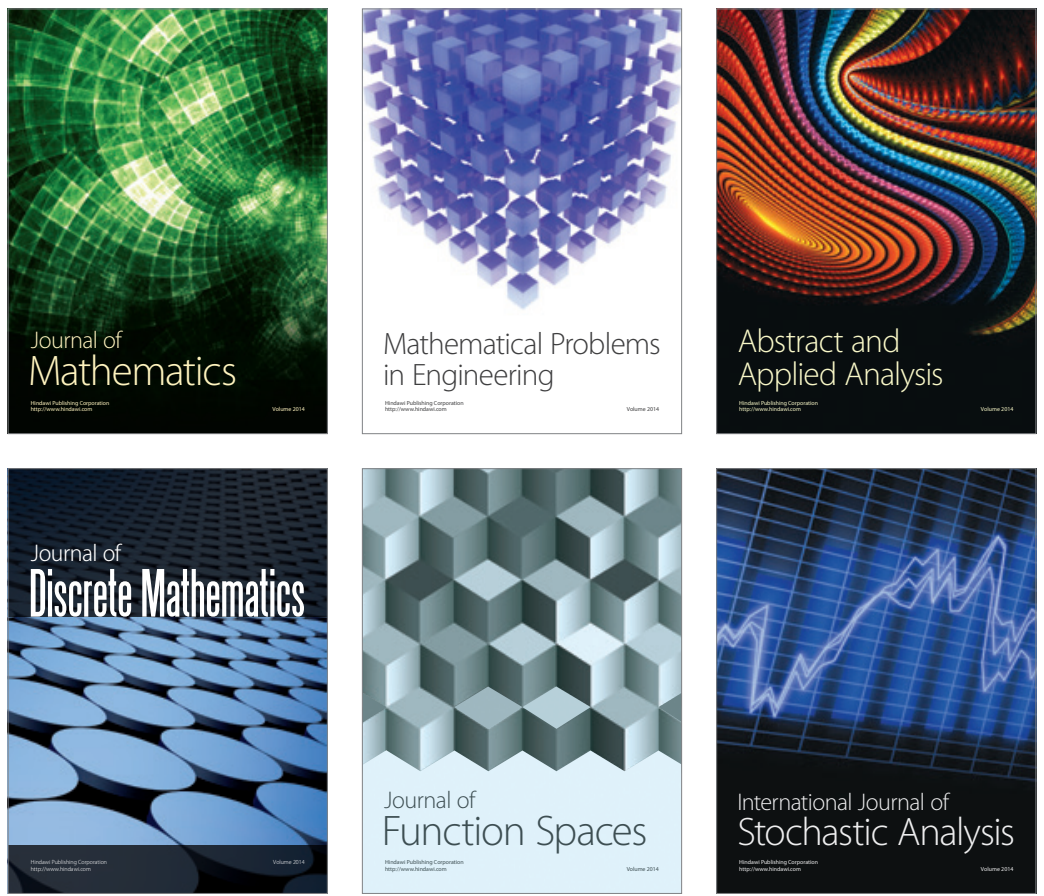

Journal of

Function Spaces

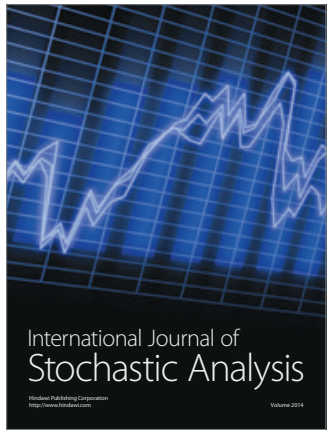

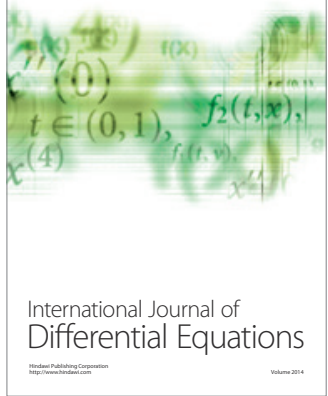
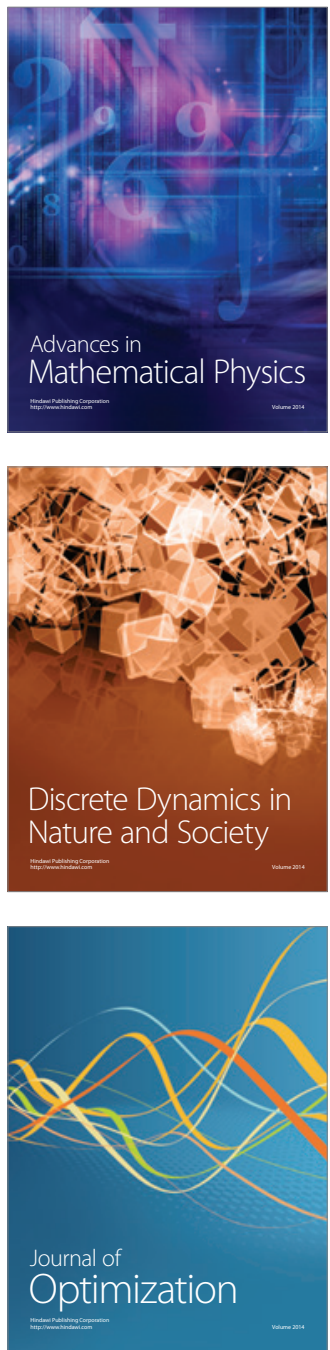